\title{
The Impact of Topological Characteristics on Ad Hoc Networks based on Complex Network
}

\author{
Dong Wang \\ College Of Information Science and Engineering \\ Hunan University \\ ChangSha, China \\ e-mail: wangd@hnu.edu.cn
}

\author{
XianYu Li \\ College Of Information Science and Engineering \\ Hunan University \\ ChangSha, China \\ e-mail: cjzlxy2012@163.com
}

\begin{abstract}
Topological characteristics is the foundation for optimizing topology structure and designing protocols. In this work, we study broadly the topological characteristics of Ad hoc networks through the perspective of complex network and identify the small-world effect, scale-free property, clustering property and hierarchy of Ad hoc networks, we also analyze the relationship among many of topological characteristics. Results shows that the degree distribution, core distribution, path distribution all present an obvious distribution trait. Moreover, the Ad hoc networks have clear small-world and clustering properties. Additionally, we found that a node whose degree is close to average degree usually has higher clustering coefficient, namely this node has a good fault tolerance and survivability.
\end{abstract}

Keywords—ad hoc networks; complex network; topological characteristics

\section{Introduction}

Ad hoc networks are self-organized, self-configured, and self-controlled infrastructure-less networks. A desirable topology can effectively increase these networks' capacity, prolong the life-time and reduce interference [1]. However, to design appropriate topology and protocols of such networks, it is key to understand the topological characteristics. But Ad hoc networks behave distinctly from others which makes the study of the topological characteristics become a challenging, interesting, and very important problem.

Complex network as an emerging interdisciplinary has aroused highly attention by all fields. It mainly concerns itself about topological characteristics. The study of networks through the complex network's perspective provides us insightful information to a deeper understanding of their natures and functions. Small-world [2] and scale-free property [3] are the two most important properties of complex network. A large number of empirical studies show that complex network widely exists in our real life, and many of real networks exhibit the same properties [4-56]. At present, the complex network has been extensively applied in the areas of physics, computer, science, biology, sociology, economic [7].

In this paper, we provide a thorough and extensive analysis of the topological characteristics of Ad hoc networks from complex network analysis. We recognize the synergy among complex network analysis and ad hoc networking as a fertile research area that can provide significant advances for the design of network, especially in topology control and network protocols. To the best of our knowledge, this is the most comprehensive study of topological characteristics of Ad hoc networks, in terms of the variety of metrics and the relationship among these metrics we consider.

The rest of the paper is organized as follows. We describe some of the works in the literature that also solve Ad hoc networks' issues through complex networks perspective in section 2. Furthermore, in section 3, the main properties of complex networks are introduced. The experimental results and analysis are shown in section 4.Finally, we discuss the primary importance of our findings for the design of Ad hoc networks solutions in Section 5.

\section{Related work}

In recent years, topological characteristics of complex network has received unprecedented attention. Literature $[8,9]$ made a fairly comprehensive overview about related concepts, research findings and applications of complex network's topological characteristics. However, existing research efforts on topology characteristics analysis of Ad hoc networks based on complex network are very few. In this section, we list some of the works that have also studied Ad hoc networks through complex networks theory.

Based on complex network theory, Rezende et al. [10 ] performed a thorough analysis of the effect of mobility in shaping the network topology in a variety of Ad hoc networks' scenarios. They analyzed the degree distribution, average path length and clustering coefficient which is the most widely used topological metrics. Results show that depending on movement behavior, a small-world state will reach earlier or later. In order to design the most effective topology, Tong et al. [11] analyzed the topological characteristics of Ad hoc networks respectively from the theory and experiment through complex network theory. They also studied the average path length, clustering coefficient, degree distribution and answered whether the Ad hoc network has small-world effect and scale-free property. By analyzing the relationship among transmission range, degree, average path length. At present, some topological metrics have been used in optimizing the topology of Ad hoc networks. Chen [12 ] proposed a topology control algorithm of wireless sensor networks under an average degree constraint. This algorithm can efficiently solve the contradiction between connectivity and sparse topology and simplify the complexity of route, and consequently, prolong the network lifetime. Brust [13]realized that network with small average path length and high clustering coefficient 
usually has high fault tolerance and communication efficiency and proposed a local small-world topology control algorithm for backbone-assisted mobile Ad hoc networks (LSWTC).

However, the previously researches on topology of Ad hoc networks just consider degree distribution, clustering coefficient, average path length. But to other topological metrics and the relationship among these metrics are not considered by any of the existing research. However, for the same network topology, the topological characteristics are varied, analyzing multiple topological characteristics is important [14].Li et al.[15]found that although the degree distribution can separate ER random networks and scale-free networks, but the degree distribution with the same power law maybe have completely different topology structure. So it is important to combine with other topological characteristic parameters in our research.

\section{Complex network topological metrics}

Before Average Path Length (APL): The shortest path length $\mathrm{d}_{i j}$ is defined as the minimum number of edges between nodes $i$ and ${ }^{j}$.The path length distribution is the probability that the path length of two randomly selected nodes has a given shortest path length.

Degree Distribution: The degree ${ }^{k_{i}}$ of node $i^{i}$ is the number of links adjacent to it. The most basic topological characterization of a network can be obtained in terms of the degree distribution $P(k)$ defined as the probability that a node chosen uniformly at random has a degree $k$.

Clustering Coefficient (CC): Suppose node ${ }^{i}$ connects with other nodes by $\mathrm{k}_{i}$ edges, these $\mathrm{k}_{i}$ nodes are neighbors of node $i$.The total number of possible edges in the neighborhood of node $i$ is $\mathrm{k}_{i}\left(k_{i}-1\right) / 2$. Let $\mathrm{E}_{i}$ be the existing edges among these $\mathrm{k}_{i}$ nodes. The local clustering coefficient $\mathrm{C}_{i}$ of node $i$ is $\mathrm{C}_{i}=2 E_{i} /\left(k_{i}\left(k_{i}-1\right)\right)$. The global clustering coefficient $C$ is then the average of all local clustering coefficient. It is also useful to consider $C(k)$, the clustering coefficient of a connectivity class $k$, which is defined as the average of $\mathrm{C}_{i}$ taken over all nodes with a given degree $k$.If $C(k)$ and $k$ has the relationship of $C(k) \sim k^{-1}$, we say that the network has a hierarchy.

Betweenness: The node betweenness equal to the number of shortest paths from all nodes to all others that pass through that node.

Core: A node has $k-$ core if and only if it belongs to the $k$ - core but not to the $(k+1)-$ core. Hence, the $k$ - core layer is the collection of all nodes having core $k$. The core of a network is the $k-$ core only when the $(k+1)-$ core is empty.

\section{Simulation and analysis}

Ad hoc networks connect wireless devices in an environment where there is no underlying infrastructure and the communications between all pairs of nodes are performed only by mutually reachable radios. When a node needs to contact a distant node that is beyond its radio range, intermediary nodes between them must be used. In this section, we discuss the model used to simulate Ad hoc networks and analyze the experimental results.

\section{A. Model and Settings}

In our experimental evaluation, we adopted one of the most widely used network models in performance analysis of Ad hoc networks. It consider a two-dimensional Ad hoc networks that consists of a set $\mathrm{V}$ of $\mathrm{n}$ wireless nodes randomly distributed in a square R2.Each node has the same maximal transmitting range and can adjust the transmission range from 0 to Rmax. We assume that all nodes have distinctive ID and are equipped with Omni-directional antennas. There is an edge $\mathrm{e}=(\mathrm{u}, \mathrm{v})$ between two nodes $\mathrm{u}$ and $\mathrm{v}$ if and only if the Euclidean distance $\|\mathrm{u}, \mathrm{v}\|$ is at most Rmax. Each node can gather its location information via GPS.

We randomly generate a set $\mathrm{V}$ of $\mathrm{n}$ wireless nodes in a $1000 \times 1000$ square and only if the original communication graph is connected can the simulation is done; the number of nodes is from 50 to 250 , the maximum transmission range $\mathrm{R}$ vary from 50 to 250 and the power constant $\beta$ is set to 2 . Each result is the average of 100 runs.

\section{B. Analysis}

Small-world and scale-free property: Small world networks have short average path length and high clustering coefficient, scale-free networks' degree distribution has a power-law distribution. In real life, the networks mainly divide into relationship networks and space networks. Small world property generally exists in relationship networks [16].but the Ad hoc networks are space networks, connections among nodes depend on the transmission range, so it is hard to have a small world property[17]. However, from figure 1 we can see that, the average path length of Ad hoc networks is less than small world network (WS), and from figure 2 we can see the clustering coefficient is much higher than random network (RG). This indicates that the Ad hoc networks have obvious small-world property. Figure 3 shows that the degree distribution of Ad hoc networks is Poisson distribution, this suggests that the Ad hoc networks does not have the scale-free property.

Path distribution: Shortest paths play an important role not only in the transport and communication within a network, but also in the characterization of the internal structure of a network. As figure 4 shows, path distribution of Ad hoc networks obeys deflective Gaussian distribution. Moreover, more than $80 \%$ of nodes' path length in the network less than 7.

Core distribution: The node which is in the core of network plays an important role in processing information transmission. Figure 5 shows that the core distribution is Poisson distribution which is similar to degree distribution. Is there some relationship between degree and core? As figure 6 shows, the core firstly grows rapidly with the increase of degree, but when the degree increases to a certain extent, it grows slowly. What is more, when the degree increases to a certain value, the core falls sharply. This indicates that a node with a big degree, may not have a big core. 
Clustering coefficient: Figure 7 shows that the relationship between $C(k)$ and $k$ is not $C(k) \sim k^{-1}$, that is to say the Ad hoc networks have no hierarchy. And combined with degree distribution, we can see the node whose degree close to average degree usually has a higher clustering coefficient .It suggests that the node who has a big degree, its clustering coefficient may be not big.

Cumulative betweenness distribution: In Ad hoc networks, the failure of a node which has a larger betweenness may cause the shortest paths become longer, and consequently, the communication efficiency will be reduced. So the betweenness for discovering and protecting critical nodes in the network is very important. From figure 8 we can see that, the accumulated betweenness distribution presents an obvious power-law distribution, this illustrates that a few of nodes have larger betweenness, but many of edges and nodes betweenness are very small.

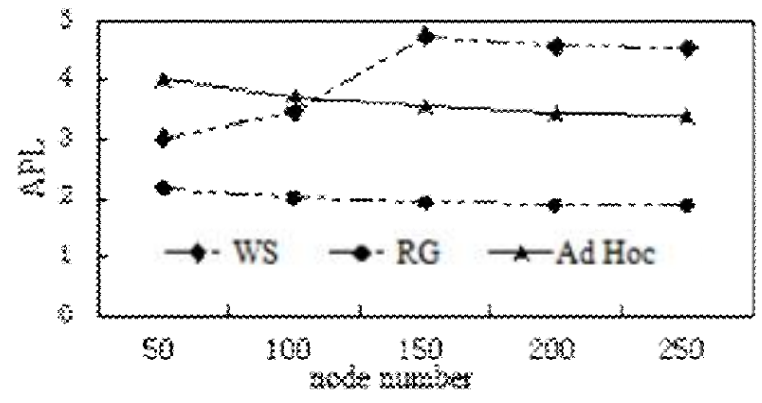

Fig. 1. Average path length of different networks

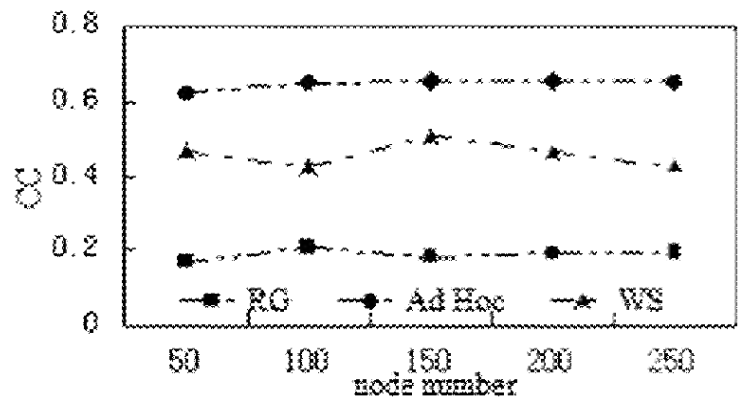

Fig. 2. clustering coefficient(right) of different networks

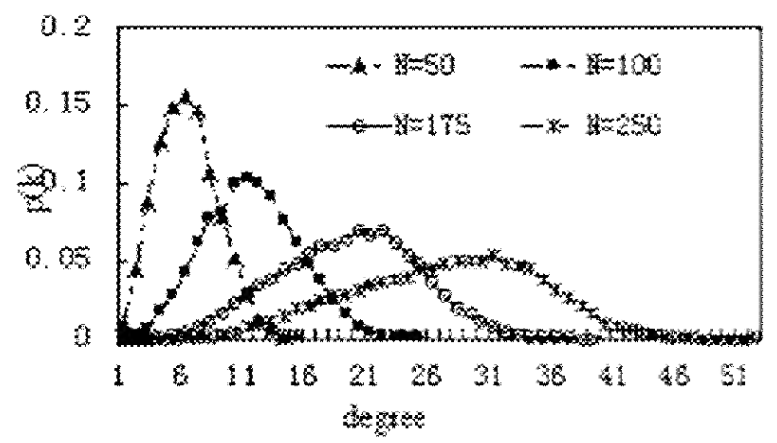

Fig. 3. Degree distribution of ad hoc networks

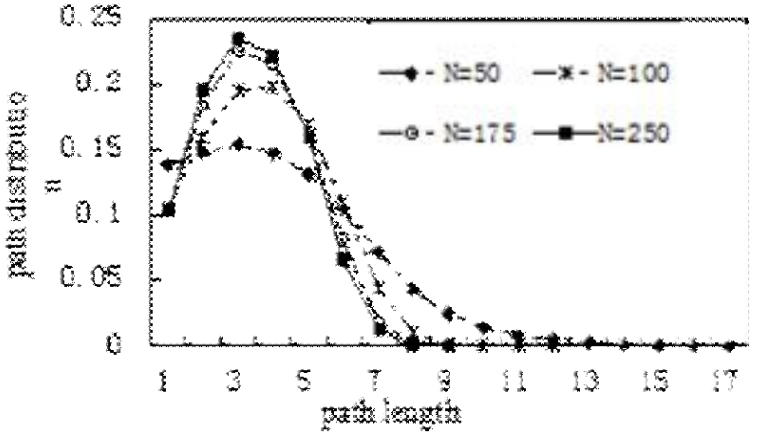

Fig. 4. path distribution of ad hoc networks

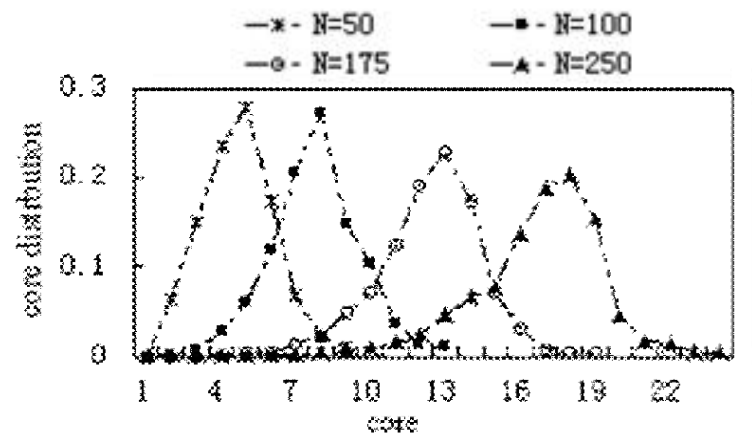

Fig. 5. Core distribution of ad hoc networks

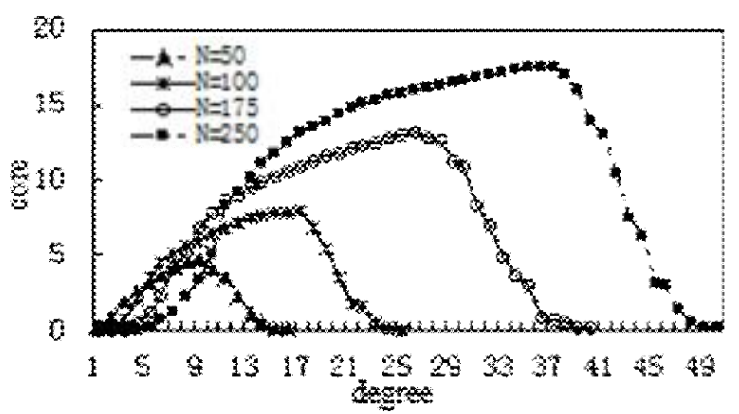

Fig. 6. The relationship between core and degree of ad hoc networks

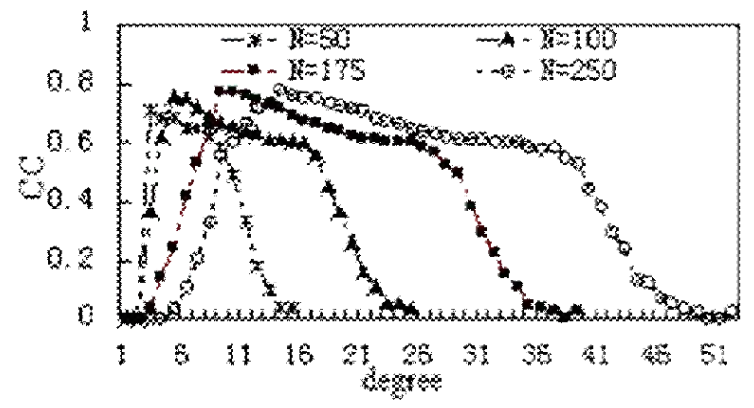

Fig. 7. The relationship between clustering coefficient and degree 


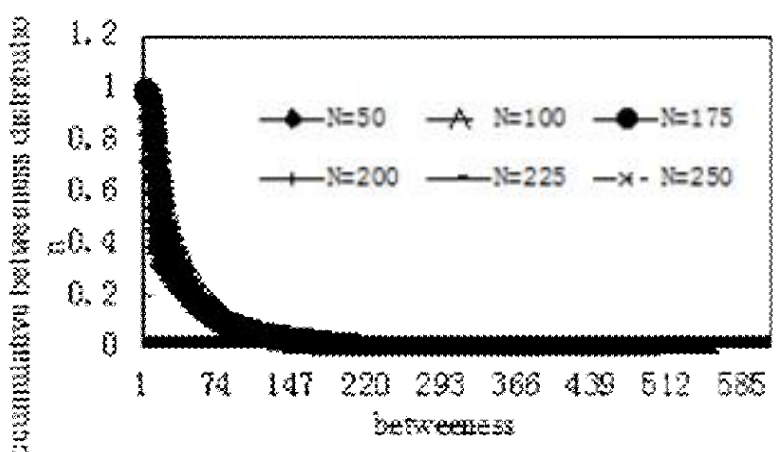

Fig. 8. Cumulative betweenness distribution of ad hoc network

\section{Conclusions}

How to optimize the topology of Ad hoc networks and provide a good underlying topology to support for the upper communication protocols has become the main content of the Ad hoc networks research. This paper we detailed the topology characteristics of Ad Hoc network based on complex network theory. Through experiment and analysis, we found that the degree distribution of Ad hoc networks is Poisson distribution, path distribution presents the deflection Gaussian distribution and the cumulative node betweenness conforms to the power-law distribution and soon on .At the same time, through the analysis of average path length, clustering coefficient, the relationship between clustering coefficient and degree of Ad hoc networks. We studied the small world property, clustering characteristics, scale-free property and hierarchy. This will provide us some useful reference for the design of Ad hoc networks protocols and topology control. We can purposefully modify the network topology structure according to the topological characteristics to improve the performance. But the network model we used is idealistic that does not take into account many problems in practical application. So we need to strengthen the research of network model that conforms to the realities .In addition, what is the relationship among these topology characteristics and how to make use of the topological characteristics to improve the network's performance also is the direction we need to study in the future.

\section{Acknowledgment}

This work was partly supported by National Natural Science Foundations of China (No. 61272061 and No.61301148), the fundamental research funds for the central universities of China (No.531107040263, 531107040276), the Research Funds for the Doctoral Program of Higher Education of China (No. 20120161120019 and No. 20130161110002), Hunan Natural Science Foundations of China (No. 10JJ5069 and No. 14JJ7023) and the Open Fund Project of Key Laboratory in Hunan Universities (No. 11K017).

\section{References}

[1] Ramanathan R, Rosales-Hain R. Topology control of multihop wireless networks using transmit power adjustment[C]//INFOCOM 2000.
Nineteenth Annual Joint Conference of the IEEE Computer and Communications Societies. Proceedings. IEEE. IEEE, 2000, 2: 404-413.

[2] Watts D J, Strogatz S H. Collective dynamics of 'small-world' networks, J. nature, 393 (1998) 440-442.

[3] Barabási A L, Albert R. Emergence of scaling in random networks, J. science, 286 (1999) 509-512.

[4] Strogatz S H. Exploring complex networks, J. Nature, 410 (2001) 268276.

[5] Albert R, Barabási A L. Statistical mechanics of complex networks, J. Reviews of modern physics, 74 (2002) 47

[6] Newman M E J. The structure and function of complex networks, J. SIAM review, 45 (2003) 167-256.

[7] Dorogovtsev S N, Goltsev A V, Mendes J F F. Critical phenomena in complex networks, J. Reviews of Modern Physics,80 (2008) 1275.

[8] Boccaletti S, Latora V, Moreno Y, et al. Complex networks: Structure and dynamics, J. Physics reports, 2006, 424(4): 175-308.

[9] Costa L F, Rodrigues F A, Travieso G, et al. Characterization of complex networks: A survey of measurements, J. Advances in Physics,56 (2007) 167-242.

[10] Rezende C, Boukerche A, Pazzi R W, et al. The impact of mobility on mobile Ad hoc networks through the perspective of complex networks, J. Journal of Parallel and Distributed Computing,71 (2011) 1189-1200.

[11] Tong C, Niu J W, Qu G Z, et al. Complex networks properties analysis for mobile Ad hoc networks, J. IET communications,6 (2012) 370-380.

[12] Chen Li-jun, et al. Topology control algorithm of wireless sensor networks under an average degree constraint, J. Chinese Journal of Computers, 30 (2007) 1544-1550.

[13] Brust M R, Ribeiro C H C, Turgut D, et al. LSWTC: A local smallworld topology control algorithm for backbone-assisted mobile ad hoc networks[C]//Local Computer Networks (LCN), 2010 IEEE 35th Conference on. IEEE, 2010: 144-151.

[14] Haddadi H. Topological characteristics of IP networks [D]. University College London, 2008.

[15] Li L, Alderson D, Willinger W, et al. A first-principles approach to understanding the internet's router-level topology[C]//ACM SIGCOMM Computer Communication Review. ACM, 2004, 34(4): 3-14

[16] Watts D J. Small worlds: the dynamics of networks between order and randomness [M]. Princeton University Press, 2001.

[17] Helmy A. Small worlds in wireless networks [J]. Communications Letters, IEEE, 2003, 7(10): 490-492. 